Dr. G. M. Collwell has been appointed instructor in mathematics at Yale University.

Professor C. E. Bikle, of Teachers College, Columbia University, has resigned to accept a position in the public schools at Syracuse, N. Y.

Mr. A. B. Frizell, of Harvard University, has been appointed professor of mathematics and astronomy in Midland College, Atchison, Kansas.

Professor F. W. Hanawalt, of Albion College, has been appointed professor of mathematics and astronomy at the University of Puget Sound.

Professor Floyd Field, of the Georgia School of Technology, has been appointed acting head of the department of mathematics in the absence of Professor O. T. GEckELER, who will spend the coming year in study at the University of Pennsylvania.

The death is announced of Israel C. Pierson, for thirtyfive years actuary of the Washington Life Insurance Company of New York. Mr. Pierson had been a member of the AMERican Mathematical Society since 1889.

Professor H. Joly, of the University of Lausanne, died August 4, at the age of 48 years.

Dr. Charles Taylor, Master of St. John's College, Cambridge, died August 12, 1908, at the age of 43 years.

\title{
NEW PUBLICA'TIONS.
}

\section{HIGHER MATHEMATICS.}

AlCayde (N.). Cálculo de probabilidades. Obra declarada de texto para la Academia de ingenieros del ejército por real orden de 23 de Octubre de 1907. Guadalajara, Colegio de Huérfanos de la Guerra, 1908. 8vo. $79 \mathrm{pp}$.

BoLzA (O.). Vorlesungen über Variationsrechnung. Umgearbeitete und stark vermehrte deutsche Ausgabe der "Lectures on the calculus of variations." In drei Lieferungen. Lieferung I. Leipzig, Teubner, 1908. 8 vo. $4+300$ pp.

M. 8.00

BURKhardt (H.). Entwicklungen nach oscillirenden Functionen und Integration der Differentialgleichungen der mathematischen Physik. Bericht, erstattet der deutschen Mathematiker-Vereinigung. (In zwei Halbbänden.) Leipzig, Teubner, 1908. 8vo. $12+3+1804$ pp.

M. 30.00 
Cantor (M.). Vorlesungen über Geschichte der Mathematik. Band IV. 5te Lieferung, Abschnitt 27 : Wallner, C. R., Totale und partielle Differentialgleichungen ; Differenzen- und Summenrechnung; Variationsrechnung. Abschnitt 28 : Cantor, M., Ueberblick über die Zeit von 1758 bis 1799. Leipzig, Teubner, 1908. 8vo. Pp. 883-1113.

DüsING (K.). Die Elemente der Differential- und Integralrechnung in geometrischer Methode dargestellt. Ausgabe A. Für Gymnasien, Realgymnasien und Oberrealschulen, sowie zum Selbstunterricht. Mit zahlreichen Uebungen. Hannover, Jäneke, 1908. 8vo. 74 pp. Cloth.

Egerer (H.). Repetitorium der höheren Mathematik. (Lehrsätze, Formeln, Tabellen.) München, Oldenbourg, 1908. 8vo. $8+351$ pp.

Emmerich (A.). Pseudogleichschenklige Dreiecke im Bereich der Winkelhalbirenden. Teil I. Mühlheim, 1908. 4to. $32 \mathrm{pp.}$

Fick (E.). Anfangsgründe der analytischen Geometrie des 4-fach ausgedehnten Raumes. (Progr.) Neuburg, 1907. 8vo. 55 pp.

Frattini (G.). La nozione d'indice e l'analisi indeterminata dei polinomi interi : lettura al IV Congresso internazionale dei matematici. Roma, Bertero, 1908. 16mo. 7 pp.

Galois (E.). See Tannery (J.).

GEIGER (K.). Zwei Kurven zweiter Ordnung in zwei- und vierpunktiger Berührung. Eine synthetischgeometrische Studie. (Progr.) Landshut, 1907. 8vo. $37 \mathrm{pp}$.

HAwLITSCHEK (K.). Integration irrationaler Differentiale. (Progr.) PragNeustadt, 1907. 8vo. $15 \mathrm{pp}$.

HiessL (M.). Einige Elemente der vierdimensionalen Geometrie. Teil II. (Progr.) Stockerau, 1907. 8vo. $17 \mathrm{pp}$.

Hırв (E.). Ueber Integraldarstellungen willkürlicher Funktionen. Erlangen, 1908. 8 vo. $66 \mathrm{pp}$.

ILNICKI (E.). Ueber die Prinzipien der Infinitesimalrechnung und über die Wandlungen, welche die Darstellung dieses Zweiges der Mathematik im Laufe seiner Entwickelung erfahren hat. (Schluss.) (Progr.) Czernowitz, 1907. 8vo. 9 pp.

JонN (F.). Ueber die kürzesten Linien auf krummen Oberflächen im allgemeinen und über die auf dem dreifachen Ellipsoide im besonderen. Teil I. (Progr.) Leitmeritz, 1907. 8vo. $21 \mathrm{pp.}$

Junker (F.). Höhere Analysis. Teil II. Integralrechnung. 3te verbesserte Auflage. (Sammlung Göschen, 88.) Leipzig, Göschen, 1908. 12mo. $190 \mathrm{pp}$. Cloth.

M. 0.80

KLUGE (W.). Besondere Systeme. Ein Beitrag zur Bestimmung von Determinanten. (Progr.) Lissa, Eulitz, 1908. 8vo. 48 pp. M. 1.50

Lenzi (F.). Primi elementi di calcolo differenziale e integrale. Livorno, Giusti, 1908 . $16 \mathrm{mo}$. $8+92 \mathrm{pp}$.

L. 1.00

Montessus (R. DE). Leçons élémentaires sur le calcul des probabilités. Philosophie du hasard, principes du calcul des probabilités, jeux de hasard, jeux savants, la spéculation, probabilité géométrique, probabilité des causes, etc. Paris, Gauthier-Villars, 1908. 8vo. $6+192$ pp. 
Nordro (A. ). Dell'inviluppo dei piani comuni a due quadriche. (Quadrispirale sviluppabile.) (Progr.) Triest, 1907. 8vo. $72 \mathrm{pp}$.

PöLr (J.). Bestimmung der Typen derjenigen viergliedrigen Gruppen in beliebig vielen Veränderlichen, die keine dreigliedrige InvolutionsUntergruppe besitzen. (Progr.) Innsbruck, 1907. 8vo. $28 \mathrm{pp}$.

Prang (C.). Determinanten. I. Hauptsätze über Determinanten. II. Einleitung in die analytische Geometrie des Raumes unter Anwendung der Determinanten. 2te Auflage. Berlin, Mayer und Müller, 1908. 8vo. $6+65$ pp. Boards.

M. 2.00

REIM (H.). Das regelmässige Dodekaeder und Ikosaeder in ihren wechselseitigen Beziehungen nach Angaben von L. Huebner. (Progr.) Schweidnitz, 1907. 4to. $28 \mathrm{pp}$.

Reisenhofer (R.). Das Apollonische Berührungsproblem in stereographischer Projektion. (Progr.) Bielitz, 1907. 8vo. 9 pp.

ScHWEFEL (A.). Betrachtungen über imaginäre Kreise. Versuch einer anderen Fassung der Möbiusschen Abhandlung. (Progr.) Brünn, 1907. 8 vo. $10 \mathrm{pp}$.

Stbiriani (F.). Formulario di matematiche ad uso degli studenti universitari. Bologna, $1908.12 \mathrm{mo}$. $350 \mathrm{pp}$.

StuRM (R.). Lehre von den geometrischen Verwandtschaften. (4 vols.) Vol. I: Verwandtschaften zwischen Gebilden erster Stufe. Leipzig, 1908. 8vo. $12+415$ pp. Cloth.

M. 16.00

Tannery (J.). Manuscrits de E. Galois. Paris, 1908. 8vo: 69 pp.

Walliner (C. R.). See Cantor (M.).

M. 2.50

WeBer (H.). Lehrbuch der Algebra. 2te Auflage. Band III : Elliptische Funktionen und algebraische Zahlen. Braunschweig, Vieweg, 1908. 8 vo. $14+733 \mathrm{pp}$.

M. 20.00

WirTh (H.). Beiträge zur Theorie der Abbildungen durch reciproke radii vectores. (Progr.) Wolgast, 1907. 8vo. $32 \mathrm{pp}$.

\section{ELEMENTARY MATHEMATICS.}

BorTolotri (E.). Nozioni pratiche di geometria e rudimenti di disegno geometrico. 4a edizione riveduta e corretta. Roma-Milano, Segati,

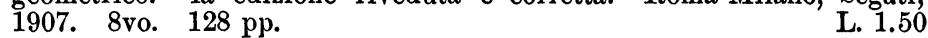

Bourlet (C.). Eléments de géométrie (géométrie plane, géométrie dans l'espace) contenant 762 exercises, rédigés conformément aux programmes de l'enseignement secondaire des garçons et de l'enseignement secondaire des jeunes filles. Paris, Hachette, 1908. 16mo. 6+382 pp. F. 2.50

Bouvart (C.) et RAtinet (A.). Nouvelles tables de logarithmes à cinq décimales. Table numérique. Tables trigonométriques. Paris, Hachette, 1908. 8vo. $176 \mathrm{pp}$.

F. 2.50

Bullard (W. G.) See Metzler (W. H.).

Cannaviello (M.). Corso di geometria elementare per le scuole medie. Parte I : Planimetria. Napoli, Priore, 1908. 8 vo. 322 pp. $\quad$ L. 2.00

CASTIE (F.). Logarithmic and other tables for schools. New York, Macmillan, $1908.12 \mathrm{mo} .36 \mathrm{pp}$.

$\$ 0.20$

Chins (M.). Lezioni di algebra ad uso dei licei. Vol. II. Livorno, Giusti, $1908.16 \mathrm{mo} .9+172 \mathrm{pp}$. $\quad$ L. 1.80 
Cortázar (D.). See Cortázar (J.).

Cortázar (J.). Tratado de geometria elemental. 35̃a edición, corregida y arreglada por D. Cortázar. Madrid, Tello, 1908. 8vo. 207 pp.

DeAkin (R.). Key to new geometry papers. London, Macmillan, 1908. $12 \mathrm{mo}$.

Gremigni (M.). Elementi di geometria ad uso delle scuole tecniche e professionali. Planimetria, libro III e IV. 3a edizione. Firenze, Bemporad, 1908 . $16 \mathrm{mo} .146 \mathrm{pp}$.

L. 0.90

HaAse (E.). See Wilk (E.).

HaMmer (E.). Der logarithmische Rechenschieber und sein Gebrauch. Eine elementare Anleitung zur Verwendung des Instruments für Studierende und für Praktiker. 4te durchgesehene Auflage. Stuttgart, Wittwer, 1908. 8 vo. $8+80$ pp.

M. 1.00

HARTL (H.). Uebungsbuch für den Unterricht in der allgemeinen Arithmetik und Algebra an Werkmeisterschulen, Baugewerkenschulen und verwandten Lehranstalten. Ausgegeben für die Schulen im Deutschen Reiche. 2te Auflage. Wien, Deuticke, 1908. 8vo. $6+161 \mathrm{pp}$.

$\mathrm{J}_{\mathrm{ACOB}}(\mathrm{J})$. Lehrbuch der Arithmetik für Obergymnasien. Wien, Deuticke, 1908. 8vo. $6+292 \mathrm{pp}$.

M. 3.20

LAISANT (C. A.). Einführung in die Mathematik. Allen Kinderfreunden gewidmet. Deutsch von F. J. Schicht. Wien, Deuticke, 1908. 8vo. $15+199 \mathrm{pp}$.

M. 2.00

LALANDe (J. DE). Tables de logarithmes, étendues á sept décimales. Nouvelle édition, augmentée de formules pour la résolution des triangles. Paris, Gauthier-Villars, 1907. $16 \mathrm{mo} .42+236 \mathrm{pp}$. 4.50

Launay (L.). Calcul de géométrie intuitive. 9e éditión. Paris, Delagrave, 1907 . $18 \mathrm{mo}$. $251 \mathrm{pp}$.

LESSER (O.). Graphische Darstellungen im Mathematikunterricht der höheren Schulen. Eine Sammlung von Materialien für die Hand des Lehrers. Leipzig, Freytag, 1908. 8vo. 108 pp.

Martin (E. ). Arithmétique, système métrique, notions de géométrie. Paris, Delagrave, 1908.16 mo. $16+356$ pp. $\quad$ F. 3.00

Martin Mengod (A.). Prolegómenos de matemáticas (nociones de aritmética y geometria). Orense, La Popular, 1907. 8vo. 176 pp.

Metzig (C.). Lehrbuch der Arithmetik und Algebra, verbunden mit einer Aufgabensammlung von mehr als 5800 Aufgaben für Baugewerkschulen, Maschinenbauschulen und andere technische Lehranstalten, für Realschulen und Mittelschulen sowie zum Selbstunterrichte. 3te vermehrte und verbesserte Auflage. Breslau, Morgenstern, 1908. 8vo. 188 pp. Cloth.

M. 2.40

Metzler (W. H.), Roe (E. D.) and Bullard (W. G.). College algebra. New York, Longmans, Green and Co., 1908. $352 \mathrm{pp.} \quad \$ 1.50$

MüLLER (H.), Die Mathematik auf den Gymnasien und Realschulen. Für den Unterricht dargestellt. Teil I. Die Unterstufe. (Lehraufgabe der Klassen Quarta bis Unter-Sekunda.) 4te Auflage. Ausgabe A. Für Gymnasien und Progymnasien. Leipzig, Teubner, 1907. 8vo. $8+136$ pp. Cloth. 
Nell (A. M.). Fünfstellige Logarithmen der Zahlen und der trigonometrischen Functionen, nebst den Logarithmen für Summe und Differenz zweier Zahlen, deren Logarithmen gegeben sind, sowie einigen anderen Tafeln. 12te Auflage. Giessen, Roth, 1908. $20+104$ pp. Cloth.

M. 2.00

Pisatr (L.). Elementi di geometria ad uso delle scuole medie inferiori. Torino, Paravia, 1907. 8vo. $5+202 \mathrm{pp}$.

L. 2.40

Ratinet (A.). See Bouvart (C.).

RICHTER (O.). Kreis und Kugel in senkrechter Projektion. Für den Unterricht und zum Selbststudium. Leipzig, Teubner, 1908. 8vo، $10+188 \mathrm{pp}$. Cloth.

M. 4.80

Row (E. D.). See Metzlar (W. H.).

Schubert (H.). Vierstellige Tafeln und Gegentafeln für logarithmisches und trigonometrisches Rechnen, in zwei Farben zusammengestellt. 3te verbesserte Auflage. (Sammlung Göschen, 31.) Leipzig, Göschen, 1908. 16mo. 128 pp. Cloth.

M. 0.80

Serret (J. A.). Traité de trigonométrie. 9e édition. Paris, GauthierVillars, 1908. 8vo. $10+336$ pp. $\quad$ F. 4.00

Threme (H.). Leitfaden der Mathematik für Gymnasien. 2 Teile. Leipzig, Freytag, 1908. 8vo. $214 \mathrm{pp.}$

M. 3.20

VAN Der Hayden (A. F). Algebraic examples. Book I. London, Arnold, 1908. 8vo. 96 pp.

$1 \mathrm{~s}$.

Villette (T. ). Eléments de géométrie pratique et de dessin linéaire, figures planes, figures dans l'espace, applications, dessin à main levée, dessin géométrique, lavis, 215 figures, 550 problèmes. Cours moyen et supérieur, rédigé conformément au programme de géométrie des écoles de Paris. 7e édition. Paris, Larousse, 1908. 8vo. $2+172$ pp.

Wells (W.). New plane and solid geometry. Boston, Heath, 1908. 12mo. $7+120$ pp. Cloth.

WilK (E.) und HAASE (E.). Anweisung zur Geometrie der Mittelschule. Teil II. Dresden, Rleyl und Kaemmerer, 1908. 8vo. 8+104 pp.

M. 1.80

\section{APPLIED MATHEMATICS.}

Amtmann (H.). Neue mathematische Theorie der Witwenversicherung. Jena, 1908. 8vo. $56 \mathrm{pp}$.

M. 2.50

BLAKE (C. F.). Mathematics of machine design with special reference to shafting and efficiency of hoisting machinery. New York, Industrial Press, 1908. 8vo. 35 pp.

$\$ 0.25$

Crew (H.). The principles of mechanics ; for students of physics and engineering. New York, Longmans, 1908. 8vo. $10+295$ pp. Cloth.

$\$ 1.50$

Hennelu (T.). Hydraulic and other tables. 3d edition. London, Spon, 1908. 8vo.

4 s. $6 \mathrm{~d}$.

ILNICKI (E.). Maturitätsaufgaben aus der darstellenden Geometrie nebst vollständigen Lösungen für die oberen Klassen der Realschulen. Wien, Deuticke, 1908. 8vo. $3+82 \mathrm{pp}$.

M. 1.25

Jouget (E.). Lectures de mécanique. La mécanique enseignée par les auteurs originaux. Part I: La naissance de la mécanique. Paris, Gauthier-Villars, 1908. 8vo. $10+210$ pp.

F. 5.00 
Kohnstamm (P.). See WaAls (J. D. van Dfr).

Kṫster (F. W.). Logarithmische Rechentafeln für Chemiker, Pharmazeuten, Mediziner und Physiker. Im Einverständnis mit der Atomgewichtskommission der deutschen chemischen Gesellschaft für den Gebrauch im Unterrichtslaboratorium und in der Praxis berechnet. 8te Auflage. Leipzig, Veit, 1908. 12mo. 107 pp. Cloth. $\quad$ M. 2.40

MACH (E.). Die Mechanik in ihrer Entwickelung historisch-kritisch dargestellt. 6te verbesserte und vermehrte Auflage. Leipzig, Brockhaus, 1908. 8vo. $18+576$ pp. Cloth.

M. 9.00

MAYER (J. E.). Das mechanische Rechnen des Ingenieurs (Rechenschieber, Rechenmaschinen, Planimeter, Integrator, Integraph). Hannover, Jänecke, 1908. $12 \mathrm{mo}$. Cloth.

M. 2.20

Newcoms (S.). La théorie du mouvement de la lune: son histoire et son état actuel. Roma, I'Únion coopérative éditrice, 1908. 8vo. $10 \mathrm{pp}$.

OrLANDI (G.). Tavola grafica tacheopantometrica per determinare colla stessa precisione delle tavole numeriche e con maggior rapidità le distanze orizzontali, le differrenze di livello, le coordinate planimetriche e le curve : instruzioni sul modo d'usare la tavola. Roma, Instituto geografico De Agostini, 1908. $16 \mathrm{mo} .15 \mathrm{pp}$.

RICHARD (G.). Mécanique revue. A l'usage des ingénieurs, constructeursmécaniciens, industriels, chefs d'ateliers et contremaîtres. $30 \mathrm{e}$ édition. Paris, Dunod et Pinat, 1908. 16mo. $6+210 \mathrm{pp}$.

F. 2.50

Rutz Castizo (J.). Tratado de mecánica racional. Vol. I : Teoria general de los sistemas de vectores ; cinemática. Madrid, Victoria-Suárez, 1908. 8 vo. $15+582 \mathrm{pp}$.

P. 18.00

SAIMOIRAGHI (A.). Instrumenti e metodi moderni di geometria applicata. Parte I: Teoria degli instrumenti misuratori, descrizione $e$ norme pratiche per l'uso. Milano, 1907. 8vo. $23+495 \mathrm{pp}$.

Sarrette (H.). Précis arithmétique des calculs̉ d'emprunts à long terme et de valeurs mobilières. Paris, Gauthier-Villars, 1908. 8yo. $19+292$ pp.

F. 10.00

WAALS (J. D. VAN DER). Lehrbuch der Thermodynamik in ihrer Anwendung auf das Gleichgewicht von Systemen mit gasförmig-flüssigen Phasen. Nach Vorlesungen bearbeitet von P. Kohnstamm. Teil I. Leipzig, Mass und Van Suchtelen, 1908. 8vo. $12+287$ pp. Cloth. 